(C) [2005] IEEE. Reprinted, with permission, from [Surya P. N. Singh and Kenneth J. Waldron, Attitude Estimation for Dynamic Legged Locomotion Using Range and Inertial Sensors, 2005, Proceedings of the 2005 IEEE International Conference on Robotics and Automation, 2005]. This material is posted here with permission of the IEEE. Such ermission of the IEEE does not in any way imply IEEE endorsement of any of the University of Technology, Sydney's products or services. Internal or personal use of this material is permitted. However, permission to reprint/republish this material for advertising or promotional purposes or for creating new collective works for resale or redistribution must be obtained from the IEEE by writing to pubs-permissions@ieee.org. By choosing to view this document, you agree to all provisions of the copyright laws protecting it 


\title{
Attitude Estimation for Dynamic Legged Locomotion Using Range and Inertial Sensors
}

\author{
Surya P. N. Singh and Kenneth J. Waldron \\ Robotics Locomotion Laboratory (LOCOLab) \\ Stanford University \\ Stanford, CA 94305, USA \\ (spns,kwaldron)@stanford.edu
}

\begin{abstract}
Legged robots offer mobility and agility in uncharted terrains. Tracking is central to legged operations and has traditionally been performed using inertial measurement techniques. The discontinuous foot fall patterns and flight phases that yield this unrivaled mobility serve to limit the motion measurement. In particular, the severe impact from repeated leg landings results in an excessive accumulation of drift. Ground range measurements, amongst several others, are robust to this drift yet are limited in application due to their low-bandwidth and variability to ground conditions.

This paper outlines the attitude estimation problem for legged locomotion, extends the ground range measurement method as an update to an inertial sensing approach, introduces the use of a hybrid estimator based on the flightphases of the legged motion, and shows preliminary results this combined approach. Based on an Extended Kalman Filter, the method takes advantage of the mostly ballistic nature of the flight phases in dynamic locomotion. Initial results indicate that the method provides fast update rates yet controls drift. In single leg experiments, which were conducted using low-cost sensing hardware, this method had an RMS error of $<0.7^{\circ}$, which was a third that of the next comparable approach. Together this provides rapid, robust estimates of flight phases and attitude necessary for extended dynamic legged operations.
\end{abstract}

\section{INTRODUCTION}

Legged locomotion is ideally suited for natural and rugged environments. Humans and other animals easily traverse rough, obstacle strewn, terrains without requiring a prepared path, as is necessary for wheeled vehicles. In order to traverse these environments efficiently and at speed, legged robotic platforms will require the use of dynamic locomotion principles [1]. Such a form of locomotion is the galloping gait of quadrupeds. The galloping gait is efficient and robust in terrain, yet is only dynamically stable. Thus, the operation and control of this rapid gait is particularly challenging as it requires an accurate estimate of robot attitude and position within this environment [2].

The rapid changes in motion associated with galloping essentially dictate that the motion measurement for dynamic legged gaits have low latency and high-bandwidth [3]. Thus, a self-contained method is desired. This is because measurements from external references, such as fixed cameras or updates from Global Position System (GPS) satellites, cannot be relied on due to the potential for occlusion, sensor eccentricities, and the extended ranges of operation needed for galloping locomotion [4]. The classical approach is to internally sense accelerations and angular motions using inertial techniques and then numerically integrate these measurements, via a deadreckoning approach, to obtain the requisite estimates. This approach has the advantage of providing rapid, high fidelity motion measurements that are robust to occlusion from external navigation aids. However, inertial navigation is an inherently difficult problem due to the inevitable drift in the estimation [5]. The long-term stability of the sensed position is diminished by integration errors, noisy readings, and offset errors. Gravitational and inertial accelerations are inseparable (for a given measurement), thus small alignment or calibration errors may appear as robot accelerations which, when integrated, results in position errors that grow quadratically in time [4]. While it is possible to regulate the drift using updates from a secondary measurement source, often with a Kalman Filter (KF), such an approach is limited in cases such as dynamic legged locomotion where many KF assumptions do not hold [6].

In dynamic legged locomotion the quadratic drift problem of inertial sensors is especially pronounced. The discontinuous foot fall patterns and phasing of legged robots, which yield its unrivaled mobility, result in repeated impacts and generate oscillations in body attitude. This acts to limit attempts at measuring this motion as the impacts excite secondary motions (i.e., mechanical vibration) which can not be readily filtered as they are often at frequencies of interest for navigation [2]. However, this vexing characteristic of dynamic legged locomotion is not without its advantages. Most legged gaits tend to be periodic and simplifying the motion provides some insight. First, [1] details a process for decomposing quadruped motion to a single-leg analog for symmetric gaits. Second, the motion of a single leg may be considered as having five phases, namely: flight, landing, compression, thrust, and lift-off [1]. The flight phase, which can be further simplified as being ballistic, is the dominant phase in which the single-leg spends most of its time [2]. Although these simplifications are idealistic and neglect key dynamic parameters, it implies that the estimation should be carried out in two parts: one for flight phase and one for ground contact [7].

Terrestrial locomotion also has the characteristic of close proximity to ground. This allows range or vision sensors to act a redundant form of position measurement. Although such methods are typically limited by sensor bandwidth, 
this approach is complementary as it is not plagued by the quadratic error growth seen in inertial approaches. Such an approach has the advantage of allowing a (exteroceptive) sensor to correct the drift from (proprioceptive) inertial sensors and further to provide gravitational vector disambiguation. The inertial sensors provide rapid updates allowing for dynamic operations and thus aid in the calibration of the external measurements.

This paper describes a procedure for gauging attitude that locates three points (via range measurements to ground), uses these points define a plane on the body of the robot, and solves for the attitude via kinematics equations. To overcome the limited bandwidth and noise of the range sensors, the estimation method uses inertial data and introduces a hybrid Extended Kalman Filter (EKF) estimator that takes advantage of the ballistic nature of flight phases in dynamic legged motion. The paper concludes by describing results from experimentally validating this method using a vertical hopping leg connected to an instrumented boom-arm platform are detailed. The approach is currently being adapted to a galloping quadrupedal robot, KOLT.

\section{KOLT PLATFORM DESCRIPTION}

The Kinetically Ordered Locomotion Test (KOLT) quadrupedal robot is part of an effort to characterize and test high-speed (up to $7 \mathrm{~m} / \mathrm{s}[25 \mathrm{~km} / \mathrm{h}]$ ) dynamic robot locomotion by galloping. Galloping is a fast, nimble gait that is preferred for quadrupeds moving at high speeds [2]. The KOLT robot (Figure 1) was designed using biomimetic principles. Unlike many research quadrupedal robots, KOLT is is of larger scale and is unique in that its twelve degrees of freedom (DOF) are fully actuated [2].

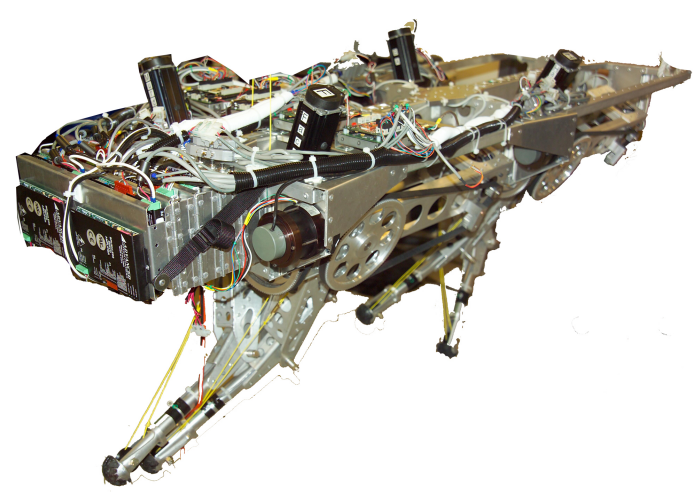

Fig. 1. The KOLT robot has been constructed specifically to study dynamic maneuvers of legged systems. The four identical legs each have three DOF and thrust primarily along the leg axis. It is approximately two meters long and weighs $70 \mathrm{~kg}$.

To propel itself and execute the maneuvers necessary for galloping, the KOLT must accurately and rapidly perform a complex series of motions. To maintain controllability and quantify the galloping motion, the on-board sensing needs to determine attitude with respect to the ground rapidly ( $i 100 \mathrm{~Hz}$ ) for extended durations (up to 10 minutes) [3]. Rapid state changes, large ground impacts, asymmetric impulses, and rapid force cycling characterize galloping locomotion. These motions, in addition to being particularly strenuous on an inertial sensing, require a robust and stiff mechanical structure as ground reaction and propulsion impulses (which are on the order of $4 g$ in nature and are nearly $9 g$ for KOLT) [2] for the entire robot must be supported instantaneously by only one leg.

\section{METHOD}

There are a variety of methods for measuring attitude. In general, the estimation problem is to determine the most likely attitude given the measurement(s) and their properties [6]. In the deterministic case, where errors are negligible, the estimate is the measurement. The algorithm presented solves for the attitude using location of at least three fixed points on the body. These points are determined relative to the ground using range measurements. This is then combined with inertial measurements via a hybrid estimator that considers whether the robot is in a flight phase to yield the final estimate of the fixed point locations and hence the attitude.

\section{A. Attitude from multiple range measurements}

The use of range data to provide a measure of orientation has been shown and is derived for an ideal planar case in [8]. This method expands on the ground range approach illustrated in [8] by using these sensors to estimate attitude and to use this data in a batch process with the inertial data. The attitude is determined for a spatial geometry as this yields a more general solution. The range equations using frame transformations (Figure 2) instead of a purely geometric approach. The orientations are solved using a pitch-roll-yaw or $\theta_{y}$-then- $\theta_{x}$-then- $\theta_{z}$ rotation sequence.

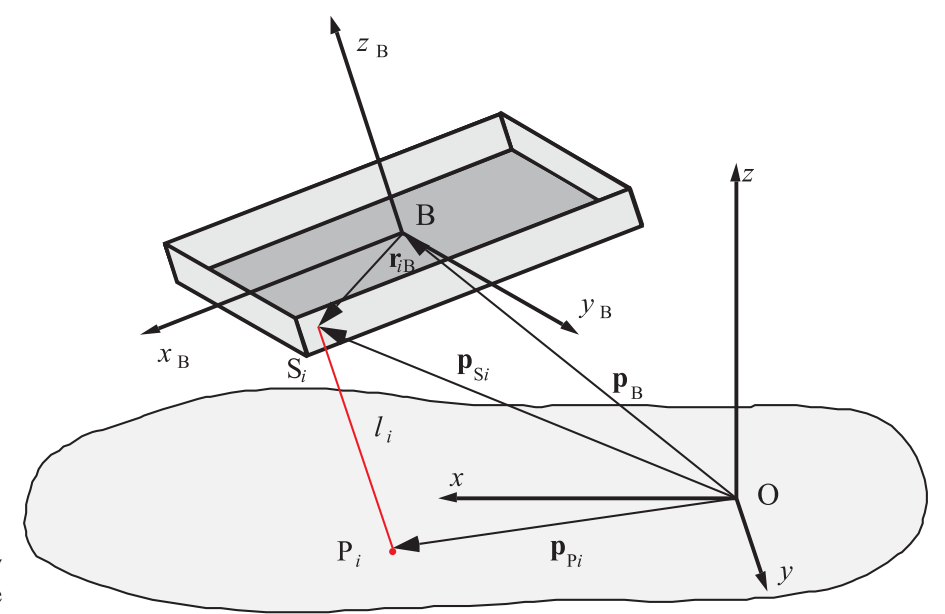

Fig. 2. Spatial estimation of attitude determination using range sensors $\left(\mathrm{S}_{i}\right.$ located by vector $\left.\mathbf{r}_{i B}\right)$ that measure the distance to point $\mathrm{P}_{i}$

Analysis of the spatial case starts by making the following assumptions: sensor positions $\left(S_{i}\right)$ with respect the body frame (B) are known and given by the vector $\mathbf{r}_{i b}=\left[\begin{array}{lll}x_{i} & y_{i} & z_{i}\end{array}\right]^{T}$, the ground is planar (i.e., the projection point $\mathrm{P}_{i}$ is in the $x y$ plane of the origin frame), that both the magnitude and angle of the sensed range are known (i.e., the vector $\mathbf{l}_{i}$ ). The location and attitude of the 
body frame with respect to the ground is represented by the position vector $p_{B}$ and the rotation matrix $\mathbf{M}$ respectively. This is solved for by taking advantage of the equivalence of the projection point $\left(\mathrm{P}_{i}\right)$ as described in both the body and fixed frames, namely that the location of $\mathrm{P}$ via the body frame $\left(\mathbf{p}_{B} \rightarrow \mathbf{r}_{i b} \rightarrow \mathbf{l}_{i}\right)$ is equivalent to its location in the fixed frame $\left(\mathbf{p}_{P i}\right)$.

By further assuming that the beam direction is perpendicular to the $x y$-plane of the body (i.e., $l_{i}$ aligned to the $\mathbf{z}_{B}$ axis), the analysis is simplified considerably. As a practical matter it is also assumed that the surface is lambertian and non-black (i.e., a visible matte surface that allows sensor to discern the range). Considering this, the position of the $i^{t h}$ sensor relative to the fixed frame can be expressed in terms of the position vector $\mathbf{p}_{S i}$ as:

$$
\mathbf{p}_{S i}=\mathbf{M r}_{i b}+\mathbf{p}_{B}
$$

where the rotation matrix, $\mathbf{M}$, is given by:

$$
\left[\begin{array}{ccc}
C \theta_{y} C \theta_{z} & -C \theta_{y} C \theta_{z} & -S \theta_{y} \\
C \theta_{x} S \theta_{z}-S \theta_{x} S \theta_{y} C \theta_{z} & C \theta_{x} C \theta_{z}+S \theta_{x} S \theta_{y} S \theta_{z} & -S \theta_{x} C \theta_{y} \\
S \theta_{x} S \theta_{z}+C \theta_{x} S \theta_{y} C \theta_{z} S \theta_{x} C \theta_{z}-C \theta_{x} S \theta_{y} S \theta_{z} & C \theta_{x} C \theta_{y}
\end{array}\right]
$$

similarly the position vector, $\mathbf{p}_{B}$, is given as:

$$
\mathbf{p}_{B}=\left[\begin{array}{lll}
x_{B} & y_{B} & z_{B}
\end{array}\right]^{\mathrm{T}}
$$

The perpendicular beam projection assumption gives that orientation of the beam $\left(l_{i}\right)$ may be represented by a unit vector parallel to the $z_{B}$ axis $\left(\mathbf{k}_{b}\right)$. Thus, the direction of the beam relative to the fixed frame is:

$$
\mathbf{k}_{r}=\mathbf{M k}_{b}=\left[\begin{array}{c}
-S \theta_{y} \\
-S \theta_{x} C \theta_{y} \\
C \theta_{x} C \theta_{y}
\end{array}\right]
$$

Thus, the position of the point $P_{i}$ in the fixed frame becomes:

$$
\mathbf{p}_{P i}=\mathbf{p}_{S i}-l_{i} \mathbf{k}_{r}=\mathbf{M r}_{i b}+\mathbf{p}_{B}-l_{i} \mathbf{k}_{r}
$$

The planar ground assumption gives that the $\mathrm{z}$ component of the $\mathbf{p}_{i}$ is zero. so:

$$
\mathbf{k} \cdot\left(\mathbf{M r}_{i b}+\mathbf{p}_{G}-l_{i} \mathbf{k}_{r}\right)=0
$$

This assumption also has the consequence of making the range measurements indeterminate to changes in yaw. Thus, $\theta_{z}$ is assumed to be zero, which gives:

$$
\mathbf{k} \cdot \mathbf{M r _ { i b }}=C \theta_{x} S \theta_{y} x_{i}+S \theta_{x} y_{i}+C \theta_{x} C \theta_{y} z_{i}
$$

thus, the governing equation for each sensor $\left(S_{i}\right)$ and measurement $\left(l_{i}\right)$ couple is:

$$
C \theta_{x} S \theta_{y} x_{i}+S \theta_{x} y_{i}+C \theta_{x} C \theta_{y}\left(z_{i}-l_{i}\right)+z_{B}=0
$$

For the three range sensors this will result in a simultaneous set of equations of the form in (8). The solution is obtained by eliminating the height $\left(\mathbf{z}_{B}\right)$ and then the roll $\left(\theta_{x}\right)$ which gives the solution for the pitch $\left(\theta_{y}\right)$ as:

$$
\begin{gathered}
N=\left(\begin{array}{c}
\left(y_{2}-y_{1}\right)\left(\left(z_{3}-z_{1}\right)-\left(l_{3}-l_{1}\right)\right) \\
-\left(y_{3}-y_{1}\right)\left(\left(z_{2}-z_{1}\right)-\left(l_{2}-l_{1}\right)\right)
\end{array}\right) \\
D=\left(y_{1}-y_{2}\right)\left(x_{3}-x_{1}\right)+\left(y_{3}-y_{1}\right)\left(x_{2}-x_{1}\right) \\
\tan \left(\theta_{y}\right)=N / D
\end{gathered}
$$

Note that a redundant configuration (in this case, more than three sensors) can be used in conjunction with the estimation process to improve robustness.

\section{B. Attitude from inertial measurements}

Inertial sensing entails the use of motion sensors, such as accelerometers and gyroscopes, whose measurements are integrated over time to monitor the body position and attitude. This is well suited for dynamic application due to the kilohertz bandwidth of the underlying motion sensors and its isolation from environmental factors. The performance is limited by noise and misalignment which leads to measurement drift. The full kinematics for six DOF inertial measurement are detailed in [5]. The discrete case may be treated effectively in state-space form as this provides a convenient method for performing the (Euler) integration [6].

An alternative approach for inertial attitude measurement on a rigid body is to instead track the location of three points by double integrating their accelerations. If the location of these points is known relative to a body centered frame, then the attitude maybe calculated via kinematic techniques similar to those shown in section IIIA. In cases where multiple accelerometers maybe placed on the body with large baselines, this approach allows for angle measurement in the cases when a gyroscope data is colored by excessive drift or simply not available. It simplifies kinematics since the position measurements, unlike attitudes, are commutative.

\section{Estimation of Attitude Via a Kalman Filter}

Given the multiple measurements sources, estimation is the process for deducing the state of the system. The Kalman filter is a linear estimation technique that provides a least-squares optimal solution to the multisensor tracking problem under several (ideal) assumptions, which include linear system dynamics and mutually independent, zeromean Gaussian (white) noise [6]. The derivation of this is detailed in [6] and demonstrated in [5] and [9].

If we define $\mathbf{x}$ as the target state vector, $\mathbf{F}$ as the system dynamics matrix, $\mathbf{H}$ as the measurement matrix, and $\mathbf{v}$ and $\mathbf{w}$ as the process and measurement noise vectors respectively; then the system can be modeled as $\dot{\mathbf{x}}=\mathbf{F x}+\mathbf{v}$, and the measurement as $\mathbf{z}=\mathbf{H} \mathbf{x}+\mathbf{w}$. Discritization of these relations is performed for a known sampling time $t_{s}$ via the fundamental matrix, $\Phi(t)$. This gives $\mathbf{F}_{k}=\Phi\left(t_{s}\right)$, where $\Phi(t)=\mathscr{L}^{-1}\left[(\mathbf{s} \mathbf{I}-\mathbf{F})^{-1}\right]$.

Similarly, the covariance of the noise vectors, $\mathbf{v}$ and $\mathbf{w}$, can be discritized to get the process and measurement noise matricies $\mathbf{Q}_{k}$ and $\mathbf{R}_{k}$ respectively. As the KF progresses in two stages (prediction and update of the state vector 
and its covariance), we introduce the notation $\mathbf{x}_{A \mid B}$ to mean the state of $\mathbf{x}$ at time $A$ based on data taken up to time $B$. The Kalman Gain, $\mathbf{K}_{k}$ scales the update between the measurement and the prediction and is derived via the Riccati equations [9]. Thus the final state estimate, $\hat{\mathbf{x}}_{k \mid k}$, is found recursively as follows [6], [9]:

$$
\begin{aligned}
\text { Prediction: } & \hat{\mathbf{x}}_{k \mid k-1}=\mathbf{F}_{k-1} \hat{\mathbf{x}}_{k-1 \mid k-1}, \\
& \mathbf{P}_{k \mid k-1}=\mathbf{Q}_{k-1}+\mathbf{F}_{k-1} \mathbf{P}_{k-1 \mid k-1} \mathbf{F}_{k-1}^{\mathrm{T}}, \\
\text { K. Gain: } & \mathbf{K}_{k}=\mathbf{P}_{k \mid k-1} \mathbf{H}^{\mathrm{T}}\left[\mathbf{H P}_{k \mid k-1} \mathbf{H}^{\mathrm{T}}+\mathbf{R}_{k}\right]^{-1}, \\
\text { Update: } & \mathbf{P}_{k \mid k}=\left[\mathbf{I}-\mathbf{K}_{k} \mathbf{H}\right] \mathbf{P}_{k \mid k-1}, \\
& \hat{\mathbf{x}}_{k \mid k}=\hat{\mathbf{x}}_{k \mid k-1}+\mathbf{K}_{k}\left(\mathbf{z}_{k}-\mathbf{H} \hat{\mathbf{x}}_{k \mid k-1}\right)
\end{aligned}
$$

\section{Legged Locomotion Extensions to the Kalman Filter}

The Kalman Filter assumptions are not very realistic for dynamic legged locomotion. This motion is unique in that it is discontinuous, the system is non-linear, and measurement noise is non-Gaussian. A general workaround is to use an EKF as it allows for nonlinear state transitions by providing a means for linearization (such as a Taylor series evaluation of $F_{k}$ ) [9]. An alternative approach is hybrid estimation with a separate estimators for each principal phases of legged locomotion. In [7] a similar hybrid estimation technique is considered for a walking machine so as to ignore acceleration as a gravity reference during ground contact.

The flight phase is the predominant mode of operation in dynamic legged locomotion. It is defined as the state when the body is freely falling [1]. This motion may be considered a ballistic trajectory and is modeled as a (nonlinear) differential equation (Eq. 11) where $g$ is the gravitational acceleration, $\rho$ is the air density, and $\beta$ is the ballistic coefficient (i.e., the extent of drag).

$$
\ddot{x}=\frac{\rho g \dot{x}^{2}}{2 \beta}-g
$$

This may be further simplified (and made linear) by neglecting the air friction. This simplification holds for low speeds and may be partially compensated for when tuning the system covariance of the Kalman filter [9]. The presence of ground contact may be determined simply by a switch or various other methods. Flight phase may be simplified as being the absence of ground contact giving the following model:

$$
\begin{gathered}
\sigma(t)= \begin{cases}1, & \text { no ground contact } \\
0, & \text { ground contact }\end{cases} \\
\bar{\sigma}(t)=(1-\sigma)
\end{gathered}
$$

This gives the following state representation (in Cartesian coordinates):

$$
\left[\begin{array}{c}
\dot{x} \\
\ddot{x} \\
\dot{y} \\
\ddot{y}
\end{array}\right]=\left[\begin{array}{cccc}
0 & 1 & 0 & 0 \\
0 & 0 & 0 & 0 \\
0 & 0 & 1 & 0 \\
0 & 0 & 0 & 0
\end{array}\right]\left[\begin{array}{c}
x \\
\dot{x} \\
y \\
\dot{y}
\end{array}\right]+\sigma\left[\begin{array}{c}
0 \\
0 \\
0 \\
-g
\end{array}\right]+\bar{\sigma}(t)\left[\begin{array}{c}
0 \\
a_{x} \\
0 \\
a_{y}
\end{array}\right]+v_{\sigma}
$$

\section{IMPLEMENTATION ON KOLT}

A design goal for the sensing system for dynamic legged locomotion is to minimize the weight, while preserving robustness to the shock from the landing leg landing. Sensor selection should also be compatible the assumptions underlying the modified EKF estimation method. That is, it simplifies estimator tuning to calibrate the sensors in advance; however, changes in calibration along with unmodeled errors may be accounted in part for by adding a bias term to the state vector.

The ground range measurement is obtained using three low-cost Sharp infrared range sensors. These miniature and mechanically robust sensors are low-weight and easily exchanged. They are not-ideal as they have large delays ( $>$ $5 \mathrm{~ms})$, low-bandwidth $(20 \mathrm{~Hz})$, variation to reflected surface color/texture, a highly non-linear response characteristic, and significant noise $(1 \mathrm{~cm} / \sqrt{\mathrm{Hz}})$. This is compensated by aggressively filtering the data; but, this has the consequence of adding nearly $50 \mathrm{~ms}$ of delay, which limits its application.

The inertial measurements on KOLT are obtained using a standard commercial IMU based on MEMS (MicroElectroMechanical Systems) accelerometers and gyros [2]. Micromachined inertial sensors, like those used on the KOLT robot, display non-linear characteristics. In particular, these sensors are marked by a predominance of $1 / f$ (or flicker) noise, offset bias, hysteresis, and misalignment. Reference [10] presents an empirically derived model for correcting addressing these factors in MEMS accelerometers. A quadratic response model, shown in Equation 15, is used to some of the non-linearity present capacitive MEMS accelerometers. In this model, $H_{1 i}$ and $H_{0 i}$ are polynomial terms obtained empirically through calibration.

$$
A_{i}\left(V_{i}\right)=H_{1 i} V_{i}^{2}+H_{0 i} V_{i}+\text { Bias }_{i} \quad[i \in(x, y)]
$$

The misalignment of the axis $(\gamma)$ was determined by checking the response at rest state when the only acceleration present is gravity. This is addressed by:

$$
\left[\begin{array}{l}
a_{x} \\
a_{y}
\end{array}\right]=\left[\begin{array}{ll}
\cos \gamma & -\sin \gamma \\
\sin \gamma & \cos \gamma
\end{array}\right]\left[\begin{array}{l}
A_{x} \\
A_{y}
\end{array}\right]
$$

While high-order low-pass filters (similar to those uses with the range sensors) can remove some of the noise, such filters add delay. This is particularly undesired for the inertial sensor. Furthermore, in order to insure proper synchronization any delay must be adjusted for when being compared to update sources, especially flight phase data. High-order filters complicate this process as the delay added is a function of the signal's frequency. Thus, the acceleration values are considered without any additional high-order filters.

Finally, flight phase timing is determined using a force sensing resistor. As detailed in [2], it is attached so as to undergo compression when the foot contacts the ground. The unit is designed with hysteresis in order to make it robust to minor perturbations. 


\section{EXPERIMENTS AND RESULTS}

This procedure was evaluated via experimental operations on a single leg connected to an instrumented, sixfoot long boom arm with two degrees of freedom (roll and yaw). For these experiments, data from the precision encoders on the boom arm, although not without error, are considered to be the actual or control values. The attitude estimates computed on the robot leg are transformed to the boom arm origin for comparison. As alluded to in the previous section, the method cannot correct for yaw in its current configuration. It is possible to use the magnetometer heading or to range to a point not on the ground plane for yaw correction.

Due to the constraints imposed by the boom arm, the linear dynamic system used by the Kalman filter was modified to include the relations present between the boom arm origin and the leg position (at the end of the arm). Here $\mathrm{x}, \mathrm{y}$ are the position estimates, $\dot{x}, \dot{y}$ are the velocity estimates, $\ddot{x}, \ddot{y}$ are the acceleration estimates, $x_{\text {bias }}, y_{\text {bias }}$ are the accelerometer biases, $a_{x}, a_{y}$ are the measured accelerations, $w$ is Gaussian system noise, $z^{\text {Range }}$ is the IR range measurement, and $v$ is the noise model for the range sensor based on its position.

$$
\begin{aligned}
& {\left[\begin{array}{c}
\dot{x} \\
\ddot{x} \\
\dot{x} \text { bias } \\
\dot{y} \\
\ddot{y} \\
\dot{y} \text { bias }
\end{array}\right]=\left[\begin{array}{cccccc}
0 & 1 & 0 & 0 & 0 & 0 \\
0 & 0 & -1 & 0 & 0 & 0 \\
0 & 0 & 0 & 0 & 0 & 0 \\
0 & 0 & 0 & 0 & 1 & 0 \\
0 & 0 & 0 & 0 & 0 & -1 \\
0 & 0 & 0 & 0 & 0 & 0
\end{array}\right]\left[\begin{array}{c}
x \\
\dot{x} \\
x_{\text {bias }} \\
y \\
\dot{y} \\
y \text { bias }
\end{array}\right]+\left[\begin{array}{l}
0 \\
1 \\
0 \\
0 \\
1 \\
0
\end{array}\right]\left[\begin{array}{l}
a_{x} \\
a_{y}
\end{array}\right]+w} \\
& {\left[z^{\text {Range }}\right]=\left[\begin{array}{lllll}
0 & 0 & 0 & 0 & 1
\end{array}\right]\left[\begin{array}{c}
x \\
\dot{x} \\
x_{\text {bias }} \\
y \\
\dot{y} \\
y \text { bias }
\end{array}\right]+v}
\end{aligned}
$$

In order to gauge the effectiveness of incorporating specific variations made to the EKF for legged locomotion, the data was also processed using a standard EKF formulation that used constant covariances for the measurement noise and did not include data from the stride period.

Experiments were performed at speeds ranging from 0 to $3.5 \mathrm{~m} / \mathrm{s}$ with an average of 100 hops per experimental run. During these runs the amount of roll and yaw was estimated using the modified EKF estimation method. As there were no measurement updates along the yaw axis, the estimator had no direct means of performing drift correction. As such, the bias estimate determined for the roll ( $y$-axis) was used instead.

The results for this method are promising and show improvement over Kalman filtered estimates, but remain colored by errors. As shown in Figures 4 and 3, the extended method described has less error compared to the control $\left(0.7^{\circ}\right.$ rms error) than the standard EKF routine $\left(1.93^{\circ} \mathrm{rms}\right.$ error). Qualitatively the proposed method is better able to estimate the full range of motions. The extent of the noise suppression resulting from the hybrid method is shown in Figure 5, which plots the raw acceleration data against values imposed by flight phase constraint (i.e., $a=\bar{\sigma} \cdot a_{\text {measured }}-\sigma \cdot g$ ).

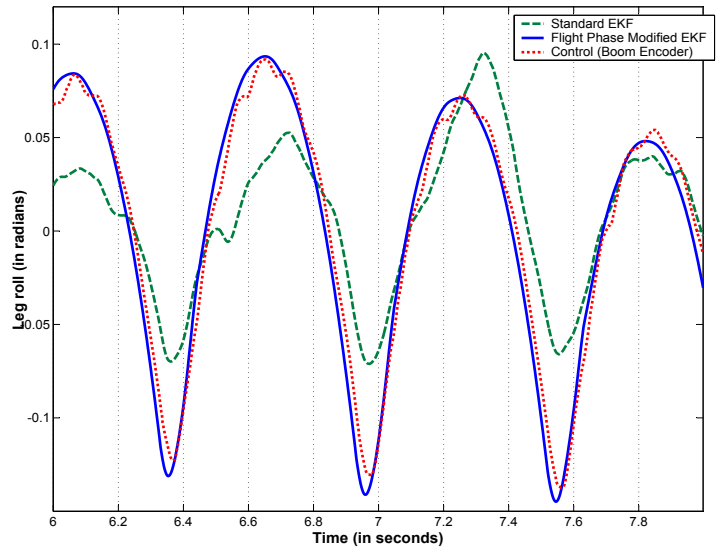

Fig. 3. Roll angle estimates for a section of the experimental run showing the slight differences between modified and standard EKF estimators. The plot shows a section consisting of four bounding periods and the attitude estimates of the two methods compared to the control value calculated from high-resolution encoders attached to the boom arm.

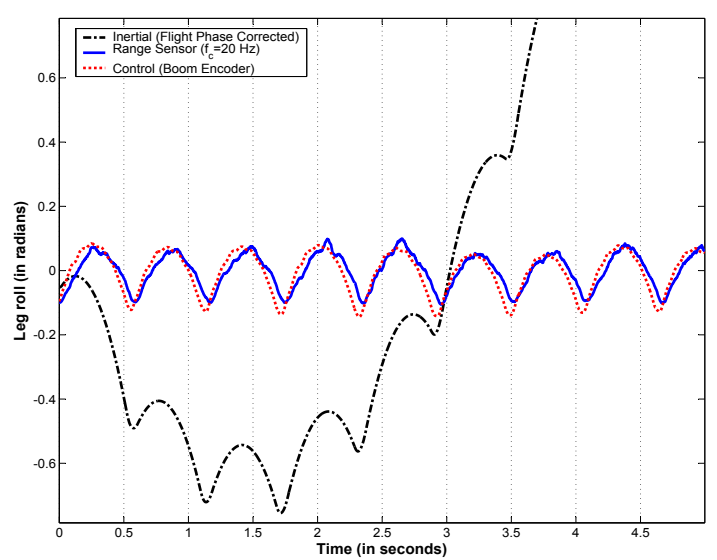

Fig. 4. Roll angle data using only the sensor data illustrates the delays present in the range measurement. Integration of the inertial measurement, even when corrected via the flight phase constraint, drifts rapidly and is greater than $30^{\circ}$ within 4 seconds.

\section{CONCLUSIONS}

Dynamic legged locomotion is a unique domain separate from aerial or wheeled locomotion. The classic solution to robot position and attitude estimation in this domain has been to use a EKF to overcome the non-linearities. This paper presents a simplified hybrid estimation architecture that is keyed to the locomotion phases. It then details and experimentally validates a hybrid method based on flight phases, which represents $81 \%$ of the operation cycle during leg boom arm experiments. 


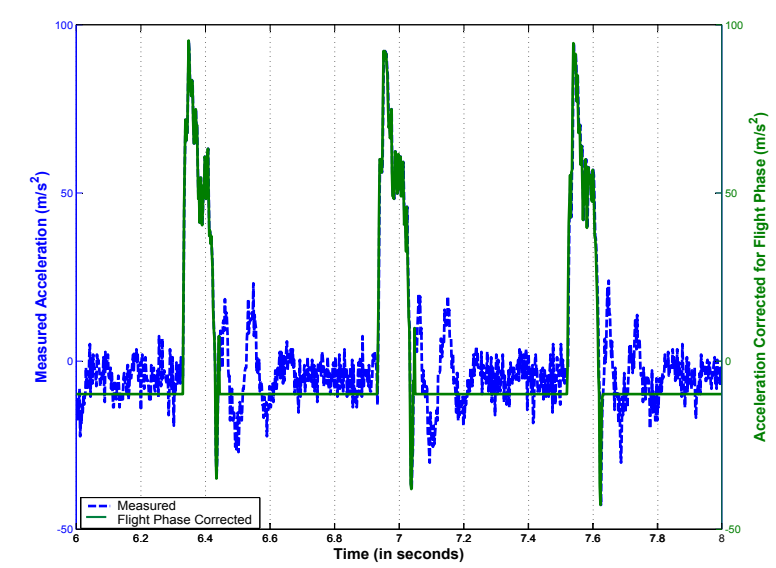

Fig. 5. Comparison between measured accelerations and acceleration data corrected such that its value is $-g$ during flight phase. The plot clearly shows the noise and cross-coupling that colors the measurement for low accelerations

Through the use of a proposed and shown that modifying EKF estimation techniques to include characteristics unique to legged robotics results in improved attitude tracking.These estimation techniques will aid in the rapid and accurate on-board estimation of attitude necessary for dynamic motion, such as galloping. The experiments performed show the use of updates from optical range measurements to be a promising means of addressing the classic quadratic drift present in inertial sensors. The infrared ranger sensors used in these preliminary experiments are very limited in applicability beyond the laboratory due to their slow performance, variance with ground reflective properties. Even with an ideal sensor, the ground based range techniques described assume a level ground plane, which thereby precludes field operations.

While it is possible to replace the infrared range sensors with laser-based range estimators, this would still be subject to geometric uncertainties and interference. Further, such an approach is still limited to planar terrains and does not provide a means to correct yaw. Ongoing experimental efforts are looking at visual odometry (and related vision routines) to determine this motion and perform the necessary updates to enable field-robust high-fidelity attitude estimations.

\section{ACKNOWLEDGMENTS}

This research and paper are supported, in part, through a National Science Foundation Grant (No. IIS-0208664) and of a National Defense Science and Engineering Graduate (NDSEG) program fellowship. The authors acknowledge the numerous contributions of the KOLT team members including J. Gordon Nichol at Stanford University and Prof. David Orin and Luther Palmer at The Ohio State University.

\section{REFERENCES}

[1] Marc H. Raibert. Legged robots that balance. MIT Press series in artificial intelligence. MIT Press, Cambridge, 1986.

[2] J. G. Nichol, S. P. N. Singh, K. J. Waldron, III L. R. Palmer, and D. E. Orin. System design of a quadrupedal galloping machine. International Journal of Robotics Research, 23(10-11):1013-1027, 2004.

[3] L.R. Palmer, D.E. Orin, D.W. Marhefka, J.P. Schmiedeler, and K.J. Waldron. Intelligent control of an experimental articulated leg for a galloping machine. In Proceedings of the International Conference on Robotics and Automation 2003, volume 3, pages 3821-3827, 2003.

[4] Jay Farrell and Matthew Barth. The Global Positioning System and Inertial Navigation. McGraw-Hill, New York, 1999.

[5] B. Barshan and H. F. Durrant-Whyte. Inertial navigation systems for mobile robots. IEEE Transactions on Robotics and Automation, 11(3):328-342, 1995

[6] Technical Staff of the Analytic Sciences Corporation. Applied Optimal Estimation. MIT Press, Cambridge, 1974.

[7] H. Rehbinder and Xiaoming $\mathrm{Hu}$. Drift-free attitude estimation for accelerated rigid bodies. In Proceedings of the International Conference on Robotics and Automation 2001, volume 4, pages 4244-4249, 2001.

[8] Dave McMordie. Towards Pronking with a Hexapod Robot. Masters, McGill University, 2002.

[9] Paul Zarchan and Howard Musoff. Fundamentals of Kalman Fltering: A Practical Approach. American Institute of Aeronautics and Astronautics, Reston, VA, 2000.

[10] W.T. Ang, S.Y. Khoo, P. K. Khosla, and C. N. Riviere. Phyiscal model of a MEMS accelerometer for low-g motion tracking applications. In Proceedings of the Interenational Conference on Robotics and Automation 2004, pages 1345-1351, 2004. 